\title{
Developing a Wearable Sensor for Continuous Tissue Oxygenation Monitoring: A Proof of Concept Study
}

\author{
Richard M. Kwasnicki, PhD, MRCS ${ }^{1}$ Ching-Mei Chen, PhD ${ }^{1} \quad$ Alex J. Noakes, BSc ${ }^{1}$ \\ Shehan Hettiaratchy, DM, FRCS ${ }^{1}$ Guang-Zhong Yang, PhD, FREng ${ }^{1}$ Ara Darzi, MD, FRCS ${ }^{1}$
}

${ }^{1}$ Hamlyn Centre, Institute of Global Health Innovation, Imperial College, London, United Kingdom

J Reconstr Microsurg Open 2021;6:e11-e19.

\author{
Address for correspondence Richard M. Kwasnicki, PhD, MRCS, 10th \\ Floor QEQM Building, St. Mary's Hospital, Praed Street, Paddington, \\ London W2 1NY, United Kingdom \\ (e-mail: richard.kwasnicki07@imperial.ac.uk).
}

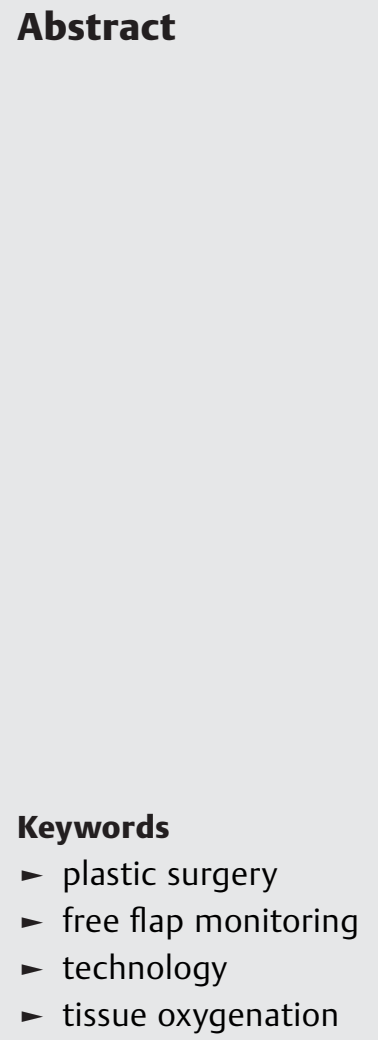

Objective Technologies facilitating continuous free tissue flap monitoring such as near infrared spectroscopy (NIRS) have been shown to improve flap salvage rates. However, the size and associated costs of such technology create a barrier to wider implementation. The aim of this study was to develop and validate a wearable sensor for continuous tissue oxygenation monitoring.

Materials and Methods A forearm ischemia model was designed by using a brachial pressure cuff inflation protocol. Twenty healthy subjects were recruited. The forearm tissue oxygenation of each subject was monitored throughout the pressure cuff protocol by using a new optical sensor (Imperial College London), and a gold standard tissue spectrometry system (O2C, Medizintecknik, LEA, Germany). Data were processed to allow quantitative deoxygenation episode comparisons between inflations and sensor modalities.

Results The correlation between $\mathrm{O} 2 \mathrm{C}$ and optical sensor oxygenation measurements was moderate (average $R=0.672, p<0.001$ ). Incremental increases in cuff inflation duration resulted in a linear increase in deoxygenation values with both $\mathrm{O} 2 \mathrm{C}$ and optical sensors, with significant differences recorded on consecutive inflations (wall shear rate, $p<0.005$ ). The presence or absence of pulsatile blood flow was correctly determined throughout by both sensor modalities.

Conclusion This study demonstrates the ability of a small optical sensor to detect and quantify tissue oxygenation changes and assess the presence of pulsatile blood flow. Low power, miniaturized electronics make the device capable of deployment in a wearable form which may break down the barriers for implementation in postoperative flap monitoring.
Free flap reconstruction is widely accepted as the gold standard management for a variety of clinical situations within plastic surgery, including open fractures and breast reconstruction. ${ }^{1}$ There is a large cost incurred from the surgery and monitoring the flap postoperatively (operation costs $£ 12,000$ and an additional $£ 45,000$ over the year). ${ }^{2-4}$ The patient themselves will bear iatrogenic stress from a donor site defect and psychological stress from aesthetic changes from surgery. Therefore, it is imperative to reduce the risk of postoperative complications.

Despite the uncontrollable patient factors that contribute to free flap failure such as age, smoking, and other comorbidities, $^{5-11}$ a potential opportunity for the improvement in received

August 26, 2020

accepted after revision

December 14, 2020
DOI https://doi.org/

10.1055/s-0041-1723995. ISSN 2377-0813.

\footnotetext{
(C) 2021. The Author(s).

This is an open access article published by Thieme under the terms of the Creative Commons Attribution-NonDerivative-NonCommercial-License, permitting copying and reproduction so long as the original work is given appropriate credit. Contents may not be used for commercial purposes, or adapted, remixed, transformed or built upon. (https://creativecommons.org/ licenses/by-nc-nd/4.0/)

Thieme Medical Publishers, Inc., 333 Seventh Avenue, 18th Floor, New York, NY 10001, USA
} 
postoperative flap surveillance could improve outcomes. Early recognition of flap failure leading to early and appropriate management can support flap salvage. ${ }^{12,13}$ Conventional clinical methods of regular flap examination for color, temperature, and other visible/palpable parameters are still seen as the gold standard. ${ }^{14,15}$ This strategy has a considerable human resource burden and has been seen to only identify $84 \%$ of failing free flaps despite high levels of clinical care. ${ }^{16}$

The most common adjunct in current clinical practice is the assessment of the vascular pedicle using a handheld Doppler probe. ${ }^{17}$ While the process of examination by handheld Doppler takes away some of the subjectivity of recognizing clinical signs, it maintains the need for in-person interaction and relies on staff being trained to use the device. These issues have been partly overcome by the use of an implantable Doppler probe, particularly the Cook-Swartz Doppler that has been shown to support free flap success rate between 95 and $98 \% .{ }^{18}$ The device does however have a high false positive rate, ${ }^{19}$ and therefore, the cost effectiveness of the device is limited due to the risk of unnecessary surgery. Cost-effectiveness may well depend on the baseline success rate of the hospital before using the device. ${ }^{3}$ Biodegradable devices which avoid the complications of wire removal from the implantable doppler are undergoing feasibility studies. ${ }^{20}$

Microdialysis is a technique established in other medical fields which involves inserting a specialized catheter into tissue to sample the biochemistry. ${ }^{19}$ Accurate measurements of glucose and lactate within buried or surface free flaps, influenced by anaerobic respiration, provide a surrogate measure of tissue oxygenation. However as of yet, there are no studies demonstrating that it is more effective than less invasive methods. Furthermore, it comes at a cost of risking local inflammation and infection caused by an inserting a foreign body, and the limitation of preexisting medical conditions or systemic factors making the results difficult to interpret. ${ }^{21}$

Near infrared spectroscopy (NIRS) utilizes the different light absorption spectra of oxy- and deoxy hemoglobin to measure tissue oxygenation. It is noninvasive and the technology is reusable, with a growing evidence base for its health care utility.

Systematic reviews report increased flap success rates using NIRS monitoring due to improved salvage rates (89 vs. $50 \%$ ) and less partial flap necrosis (15 vs. $80 \%$ ) in metaanalyses of over 1,500 free flaps. ${ }^{22,23}$ Also, a large 10-year case series by Koolen et al exhibits the benefits of NIRS, demonstrating flap salvage rates improving from 57.7 to $96.6 \%$, leading to a reduction of flap loss from 2.9 to $0.1 \%$ $(p<0.001) .^{24}$ The same review concluded that uptake of the technology is held back by the requirement for specialist training or technical support from a biomedical engineer, which is likely to incur costs on top of the often prohibited initial cost of the hardware.

The ideal free flap monitoring technology was described by Creech and Miller in 1975: "Should be harmless to patient and flap, rapidly responsive, accurate, reliable, and applicable to all types of flap. Furthermore, it should be equipped with a simple display so that even relatively inexperienced personnel can alert the development of circulatory impairments."25
A novel tissue oxygenation monitoring device (referred to as the "optical sensor") was developed based on the principals of spectroscopy with the potential for wearable form to overcome the challenges and limitations of current technologies. ${ }^{26,27}$

The aim of the study was to validate the miniaturize optical technology in measuring local tissue oxygenation by using a custom-made brachial pressure cuff forearm ischemia model. Measurements were compared with a well-validated tissue spectroscopy system (O2C, Medizintecknik, LEA, Germany).

\section{Materials and Methods}

\section{Optical Sensor Development}

A miniature $(19.6 \times 12.4 \mathrm{~mm})$ sensor was developed to continuously monitor tissue oxygenation and pulsatile blood flow. The device is made from ultra-low power electronics which consume $6.6 \mathrm{~mW}$ (in contrast to most other medical devices which are over $1,000 \mathrm{~W}$ ). The sensor detects backscattered light from two light sources onto a printed circuit board, similar to NIRS; both utilize the difference in absorption between oxygenated and deoxygenated hemoglobin. However, the wavelengths used for our low power device (740 and $880 \mathrm{~nm}$ ) are slightly out of the typical NIRS spectra. Sensor data calibration and trials of different light wavelengths resulted in a usable algorithm, which provided a tissue oxygenation estimate covering a range of oxygen saturations. This simple but effective optical sensor has energy demands easily met by a small battery making the technology capable of wireless data transmission.

All of the hardware for data collection and processing is on the wearable part of our sensor, whereas for most NIRS or other wired devices, the processing/communication hardware is inside a separate computer or bedside console. This allows our sensor to be fully wireless.

However, a wired connection was maintained throughout for the ease of data collection and practicality, as wireless performance was outside the scope of the sensor laboratory validation (-Fig. 1).

\section{Forearm Ischemia Model}

A forearm ischemia model was designed to simulate the changes in free flap failure by using a brachial pressure cuff inflation protocol in healthy subjects. A commercially available spectroscopy system $(\mathrm{O} 2 \mathrm{C})$ was used to develop and validate the protocol to produce consistent, incremental changes in forearm tissue oxygenation. The $\mathrm{O} 2 \mathrm{C}$ consists of a computer and wired probe, mounted on a trolley with approximately $1 \mathrm{~m}^{2}$ footprint. Parameters are measured at two tissue depths by using the absorption spectrum of white and laser light and include tissue oxygen saturation, relative hemoglobin (rHb, i.e., microvasculature filling pressure, raised in venous congestion), and blood flow by using parallel laser Doppler. The O2C tissue oxygen component has been validated by using polarographic pO2 measurements from a microcatheter directly inserted into an animal free flap model. ${ }^{28}$ The $02 \mathrm{C}$ has been implemented in free flap monitoring ${ }^{29}$ and numerous other clinical applications and research protocols including investigating tumor biology, ${ }^{30}$ 


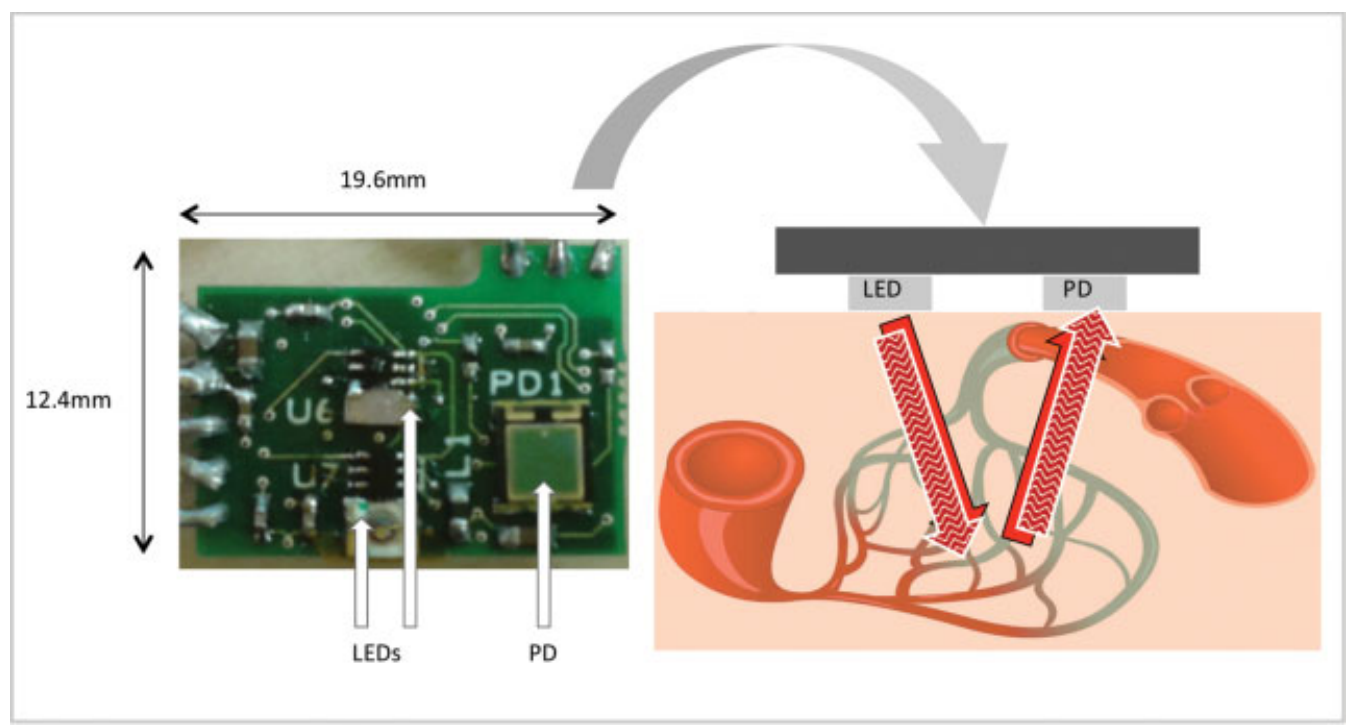

Fig. 1 Optical sensor platform and schematic showing printed circuit board and tissue interrogation method via measuring changes in backscattered light following absorption in the microvasculature. LED, light emitting diode; PD, photodiode.

pathophysiology in the critical care setting, ${ }^{31}$ musculoskeletal and sports medicine, ${ }^{32}$ and wound-healing processes..$^{33}$

Superficial $(3 \mathrm{~mm})$ forearm tissue oxygenation was more reactive to pressure cuff inflations than deep $(7 \mathrm{~mm})$, making it a more ideal comparator for the study. An arterial occlusion (AO) model was simulated by using supra-systolic cuff inflation, which caused a linear reduction in oxygenation before a plateau at low levels. A venous congestion (VC) model was simulated by using subdiastolic cuff inflation, which caused a slower reduction in oxygenation with a rise in the relative hemoglobin measurement (reflecting tissue congestion). Blood flow was maximally and partially reduced during AO and VC models, respectively, as shown by Doppler measurements. A series of five cuff inflations of incremental duration from 30 to 150 seconds was chosen based on methodological suitability and minimizing subject discomfort (—Fig. 2).

\section{Healthy Subject Study}

Ethical approval was granted from the NHS South East London Research Ethics Committee 3 (reference no: 10/H0808/124). Adult subjects were recruited, given an information sheet and the opportunity to ask any questions. Upon agreement to participate, written consent was taken by the researcher.

Each subject was seated with their left arm resting pronated on a table at the level of the heart (-Fig. 3). A manually operated brachial pressure cuff was placed on the upper arm. The optical sensor and $\mathrm{O} 2 \mathrm{C}$ probe were placed on the extensor surface of the mid forearm parallel to each other in the longitudinal axis. During placement, care was taken to avoid visible or palpable superficial vasculature, while maintaining maximum distance between the sensors to minimize "cross talk" from sensor light sources. An opaque adhesive dressing was used to stabilize the sensor placement as well as control the ambient light conditions.

A flat $\mathrm{O} 2 \mathrm{C}$ probe (LF-2) was connected to a $\mathrm{PC}$ running a standard monitoring protocol on the $\mathrm{O} 2 \mathrm{C}$ software. The optical sensor was wired via USB to a PC running bespoke software. Both software packages allowed real-time data interrogation and estimation of tissue oxygen saturation, facilitating sensor repositioning and/or repeated cuff inflations upon artifactual measurements (following ambient light calibration). A 1 to 2 minutes data stabilization phase was conducted upon initiating data collection before commencing the cuff inflation protocol. Stabilization (not necessarily return to baseline) of metrics was observed before subsequent cuff inflations and upon completion.

\section{Data Analysis}

Data from the $\mathrm{O} 2 \mathrm{C}$ and optical sensor were exported and formatted to align the oxygenation measurements to facilitate statistical analysis and comparison. The correlation of hundreds of optical sensor oxygenation measurements to that of the $\mathrm{O} 2 \mathrm{C}$ was assessed to determine the extent of concurrency. Due to data being monotonic and polynomially distributed, Spearman's Rho correlation coefficients were calculated for both general data trends, as well as tissue changes during the cuff inflation protocol for each subject. Pre- and postprotocol metrics were compared by using Wilcoxon's signed rank tests. For comparisons regarding samples considered independent, Mann-Whitney U tests were implemented.

\section{Results}

Between April 2013 and November 2013, 20 healthy subjects were recruited. All subjects completed the cuff inflation protocol while wearing both sensors.

Oxygen saturation values from the $\mathrm{O} 2 \mathrm{C}$ machine and the optical sensor showed similar trends during the cuff inflation protocol. The correlations between $\mathrm{O} 2 \mathrm{C}$ and optical sensor oxygenation measurements were moderate and statistically significant for all subjects, with Spearman's rho values ranging from 0.418 to 0.862 with an average of 0.672 $(p<0.001 ;$ - Fig. 4). 


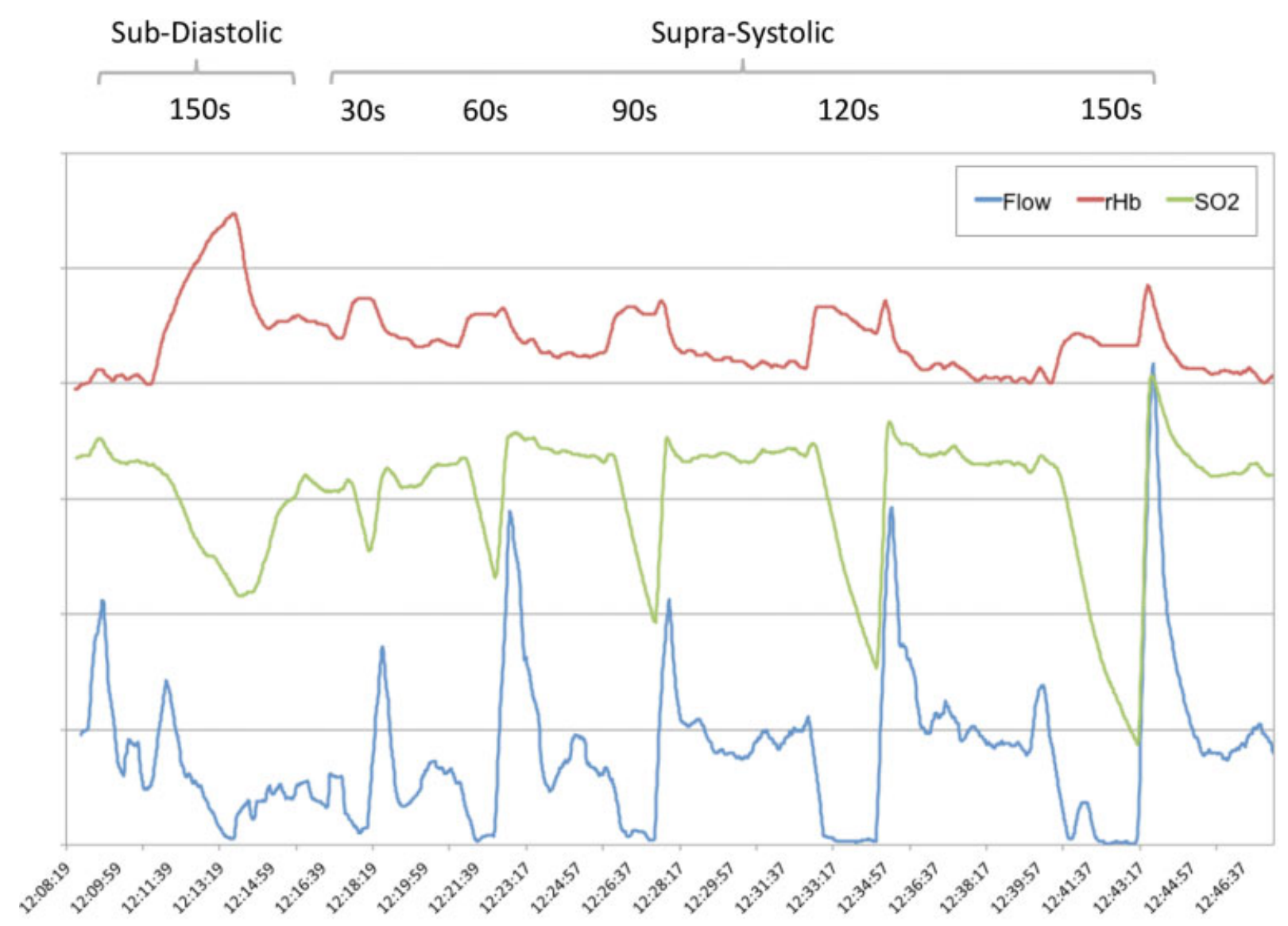

Fig. 2 Graph shows 3 metrics from the $\mathrm{O} 2 \mathrm{C}$ equipment to validate the final pressure cuff protocol. During each cuff inflation, blood flow (blue) reduces before short period of hyperaemia, tissue oxygenation saturation (green) reduces, and tissue congestion (red) increases slightly.

Incremental increases in $\mathrm{AO}$ model duration resulted in a linear increase in deoxygenation values with both $\mathrm{O} 2 \mathrm{C}$ and optical sensors. There was a significant difference in the extent of deoxygenation between subsequent cuff inflations $(p<0.005$; - Table 1 and - Fig. 5$)$.

The VC model caused a visible change in the forearm skin color and vascular prominence (-Fig. 6). The magnitude of deoxygenation as measured by the $\mathrm{O} 2 \mathrm{C}$ was less during the VC model compared with the AO model (57.9 vs. 82.2, $p=0.001$ ); however, the optical sensor recorded similar deoxygenation both models ( 23.1 vs. $18.1, p=0.099$ ).

\section{Deoxygenation Curves}

The morphology of deoxygenation curves seen during AO showed intersubject variation. Some subjects exhibited "sharp" troughs, that is, linear deoxygenation until cuff deflation, followed by immediate reoxygenation, whereas others were "softer" with an apparent plateau before cuff deflation. This pattern was mirrored in both optical and O2C datasets (-Fig. 7).

Tissue Congestion and Blood Flow Data Trends (O2C Only) Forearm tissue congestion (as measured by relative $\mathrm{Hb}$ ) was significantly greater in the VC model compared with the AO model over 150 seconds ( 27.1 vs. 9.3, $p=0.001$ ). Reduction in blood flow during the AO model was $100 \%$ after 60 seconds. In comparison, the VC model showed significantly less reduction in blood flow (74.4 vs. $98.6 \%, p<0.001)$. There was no difference in baseline and post-protocol blood flow (11.2 vs. $11.7, p=0.323$ ).

\section{Optical Sensor Detection of Pulsatile Blood Flow}

At rest, the alternating component of the optical sensor signal showed a coefficient of variation $(\mathrm{CV}=$ standard deviation/mean) of approximately 0.03 , caused by pulsatile blood flow. During the AO model, the variation was reduced to less than 0.01 , reflecting absence of pulsatile flow. The original signal resumed following cuff deflation (-Fig. 8).

\section{Discussion}

This study describes and evaluates a useful healthy subject model for free flap failure and provides laboratory validation of a low-power simplified tissue oxygenation sensor.

Changes in tissue oxygenation were simulated by using a pressure cuff model. In addition to oxygenation values, the $\mathrm{O} 2 \mathrm{C}$ provided measurements of tissue congestion ( $\mathrm{rHb}$ ) and blood flow within sampled tissue. The AO model, simulated by supra-systolic cuff inflation caused a triad of rapidly decreasing tissue oxygenation, slightly elevated tissue congestion and complete cessation of blood flow. Conversely, the VC model, simulated by subdiastolic cuff inflation, caused a more slowly decreasing tissue oxygenation, significant tissue congestion and a reduced, yet present, blood flow. Therefore, the 


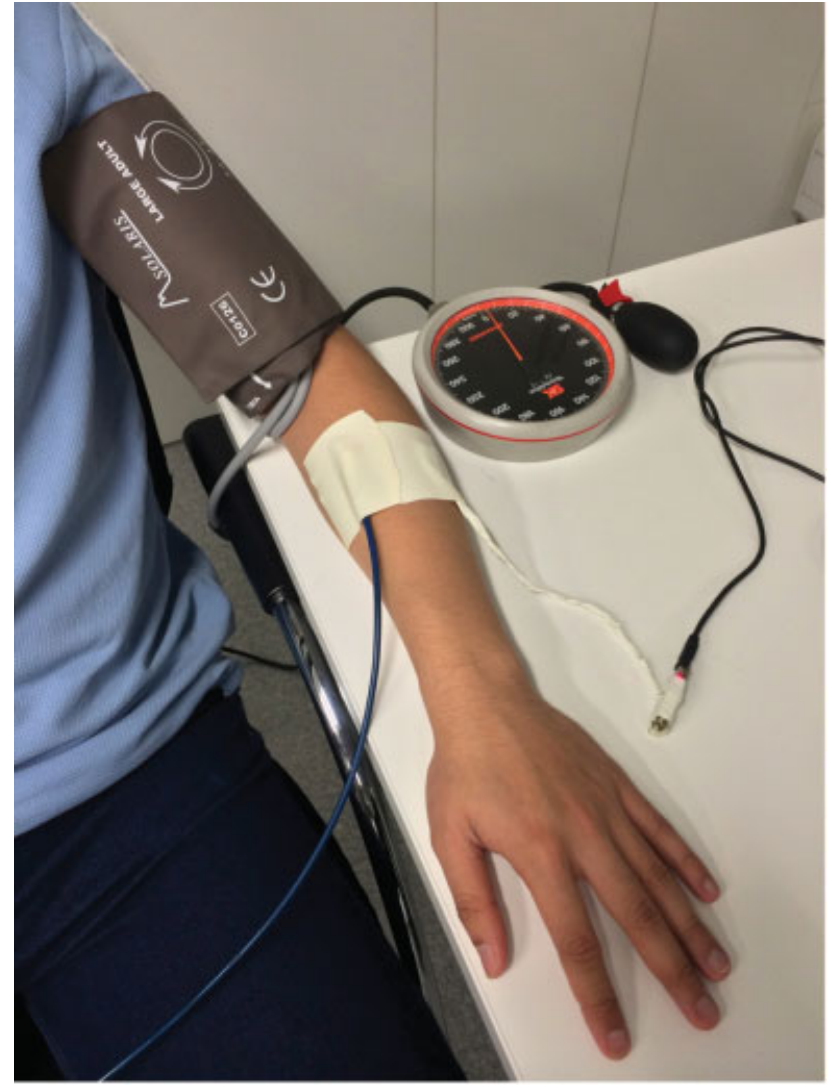

Fig. 3 Experimental setup. Manual brachial pressure cuff on the upper arm, O2C probe on radial side, optical sensor on ulnar side of forearm, both wired to PCs. cuff protocol was consistent, clinically representative, and provided the ideal model for validating the new optical sensor.

Using the protocol described, the optical sensor proved capable of detecting and quantifying changes in superficial tissue oxygenation, as compared with the current gold standard equipment (O2C). However, the relative oxygenation changes from the optical sensor were smaller, with a proportionally larger standard deviation compared with the O2C. Furthermore, the optical sensor recorded equal deoxygenation for $\mathrm{AO}$ and $\mathrm{VC}$ models, in contrast to the $\mathrm{O} 2 \mathrm{C}$ which found greater deoxygenation during the AO model. While this does not reduce clinical utility, it does highlight the balance of benefits between sensing modality, in which to achieve a low power, miniaturized device there will be limitations in the level of complexity of information recorded. Similar results were seen in 20 subjects of different gender, age, and skin color, proving external (in addition to internal) validity. The study also met the secondary aim of detecting pulsatile blood flow. This feature complements tissue oxygenation measurements by suggesting the mechanism of deoxygenation, differentiating between arterial and venous occlusion or congestion, therefore acting as a substitute for the $\mathrm{O} 2 \mathrm{C}$ congestion ( $\mathrm{rHB}$ ) and flow measurements. While the low power design may make the optical sensor less sensitive to changes, the deoxygenation episodes were still reliable and quantitative and most likely sufficient to detect a failing flap. As such, a balance must be struck between use of additional power for signal amplification to improve response and power conservation to optimize battery life.

The results of this study support the theory that a miniaturized wearable sensor could be used to detect or

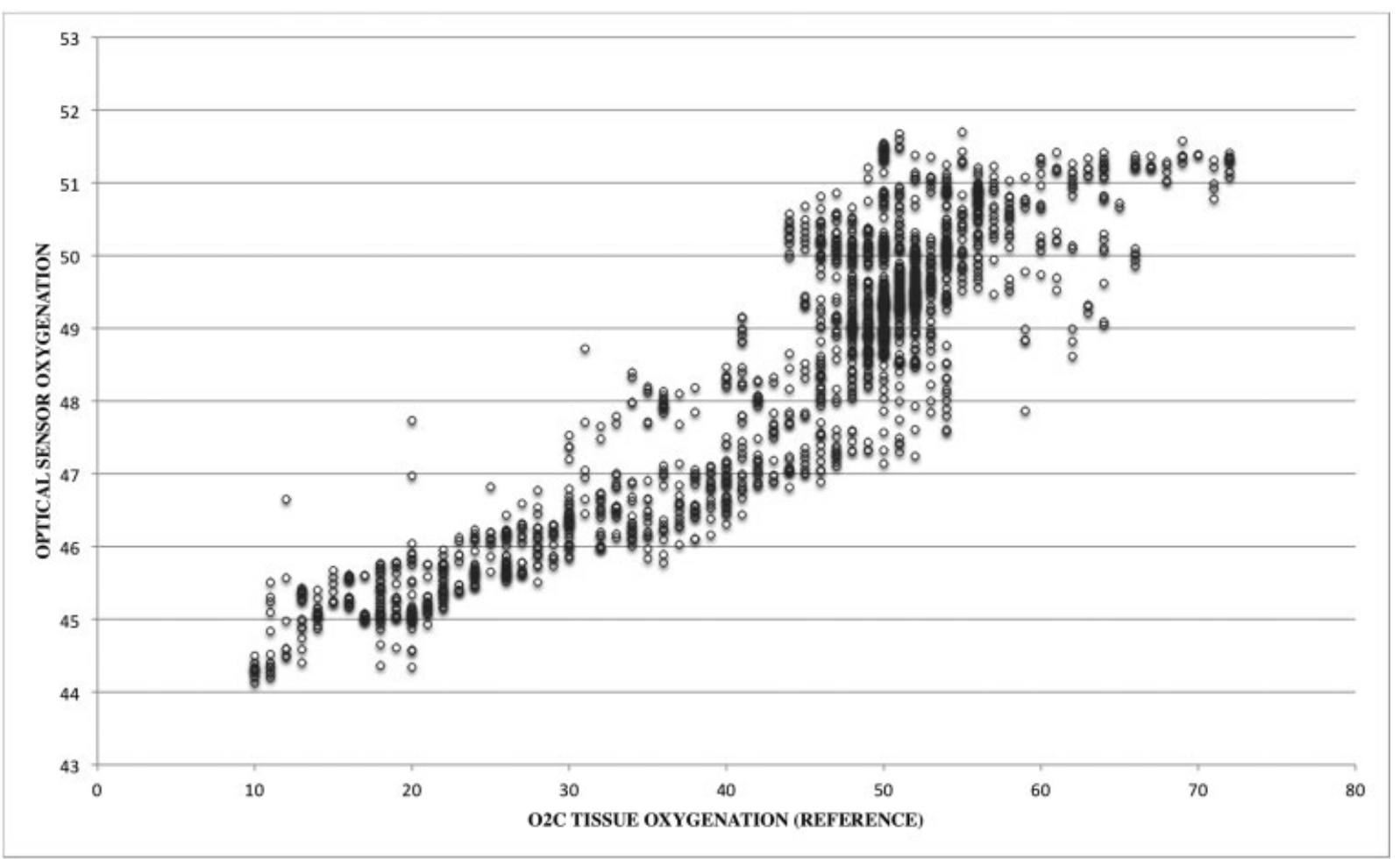

Fig. 4 Comparative data from optical sensor and $\mathrm{O} 2 \mathrm{C}$ for one subject shows strong positive correlation in tissue oxygenation during the cuff protocol. 
Table 1 Oxygenation saturation reduction (percentage) during arterial occlusion model and statistical difference between subsequent inflation durations

\begin{tabular}{|l|l|l|l|l|l|l|l|l|l|}
\hline Duration (s) & $\mathbf{3 0}$ & $p$-Value & $\mathbf{6 0}$ & $p$-Value & $\mathbf{9 0}$ & $p$-Value & $\mathbf{1 2 0}$ & $p$-Value & $\mathbf{1 5 0}$ \\
\hline O2C & 36.9 & 0.001 & 55.5 & 0.001 & 67.5 & 0.003 & 76.5 & 0.005 & 82.2 \\
Mean (SD) & $(9.80)$ & & $(5.8)$ & & $(4.0)$ & & $(6.8)$ & & $(7.7)$ \\
\hline Optical & 6.77 & 0.002 & 10.15 & 0.001 & 12.96 & 0.004 & 15.82 & 0.004 & 18.10 \\
Mean (SD) & $(2.33)$ & & $(2.73)$ & & $(2.96)$ & & $(3.50)$ & & $(3.23)$ \\
\hline
\end{tabular}

Abbreviation: SD, standard deviation.

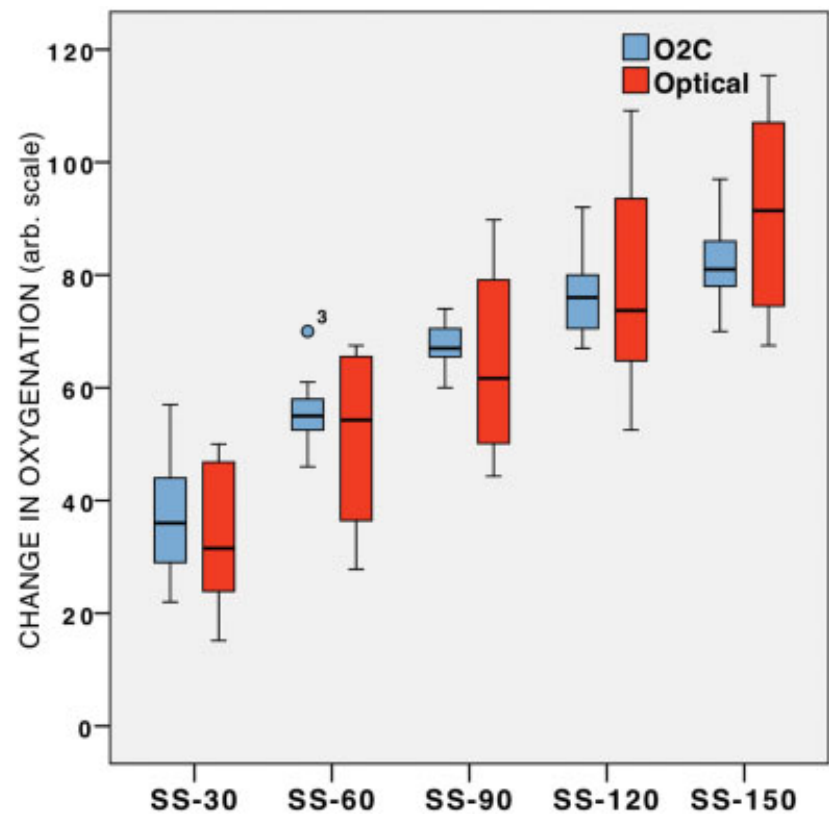

Fig. 5 Change in tissue oxygenation values during incrementally longer cuff inflations for optical sensor and $\mathrm{O} 2 \mathrm{C}$ predict flap compromise. There is already evidence whereby similar technologies have been used in clinical trials to accelerate the detection of flap failure and consequently enhanced flap salvage rates. ${ }^{24,34,35}$ Many of the currently available flap monitoring equipment require both a wired probe and an expensive bedside unit. Due to all the components being located on the patient, this device would not require a bedside unit. This reduces upfront equipment costs and improves the access of flap monitoring in smaller centers or those with multiple simultaneous free flap cases. With its wireless capability, this sensor could remain in place while the patient is mobilizing on the ward or during flap training protocols (e.g., dependency). Additionally, it avoids the needs and associated risks of invasive devices such as implantable Doppler.

As demonstrated by other sensors based on the body sensor network architecture, this device not only monitors tissue oxygenation, but also may provide a continuous data stream to appropriate staff members at remote locations. This gives rise to opportunities including push notifications to surgeons to recommend inspection of the flap, or overnight data feeds to relieve the on-call team or nursing staff from regular inspections of the flap and disrupting the patient. This could be implemented through a secure phone application or web page.

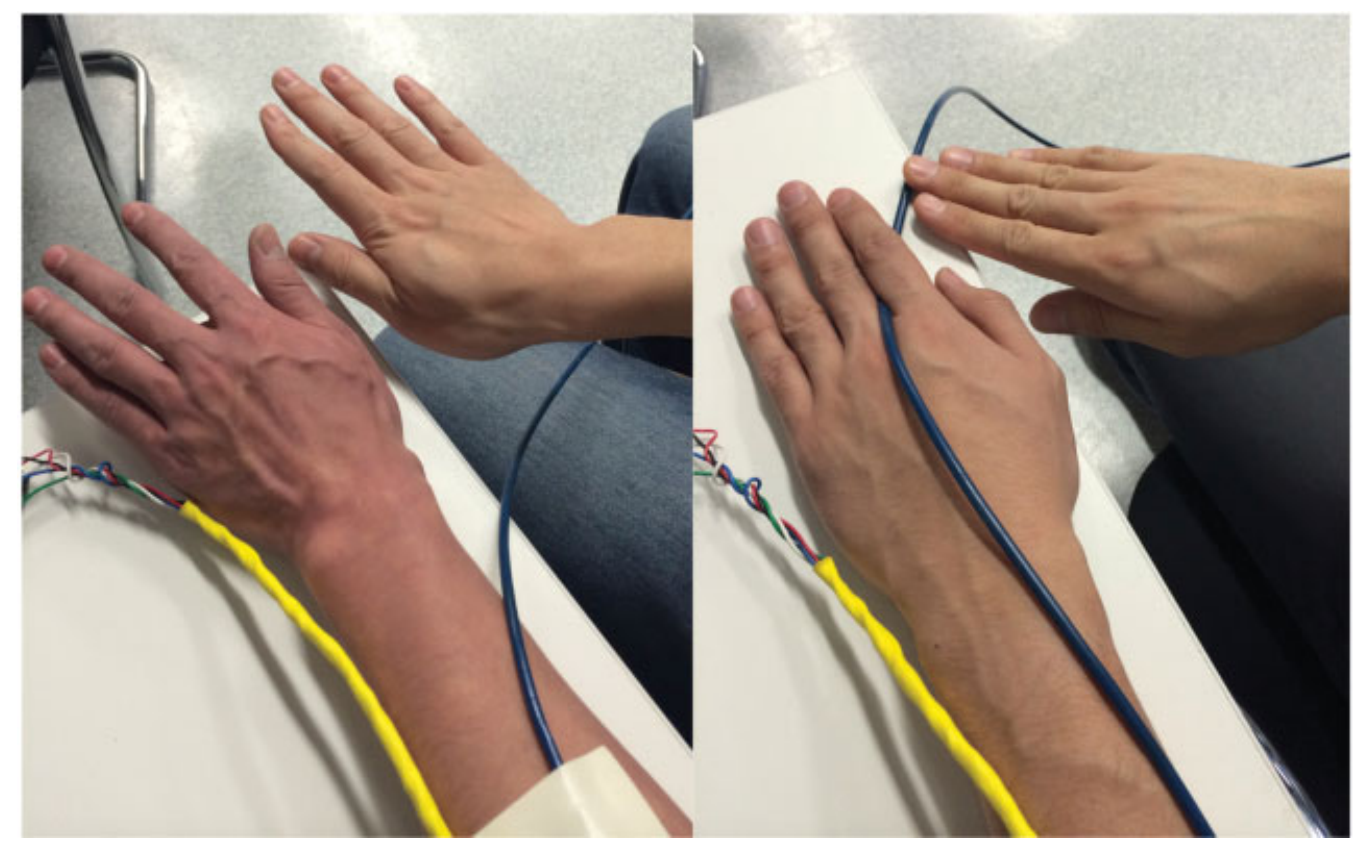

Fig. 6 Hand and forearm appearance 2 minutes 30 seconds after venous congestion model (left) and arterial occlusion model (right). 


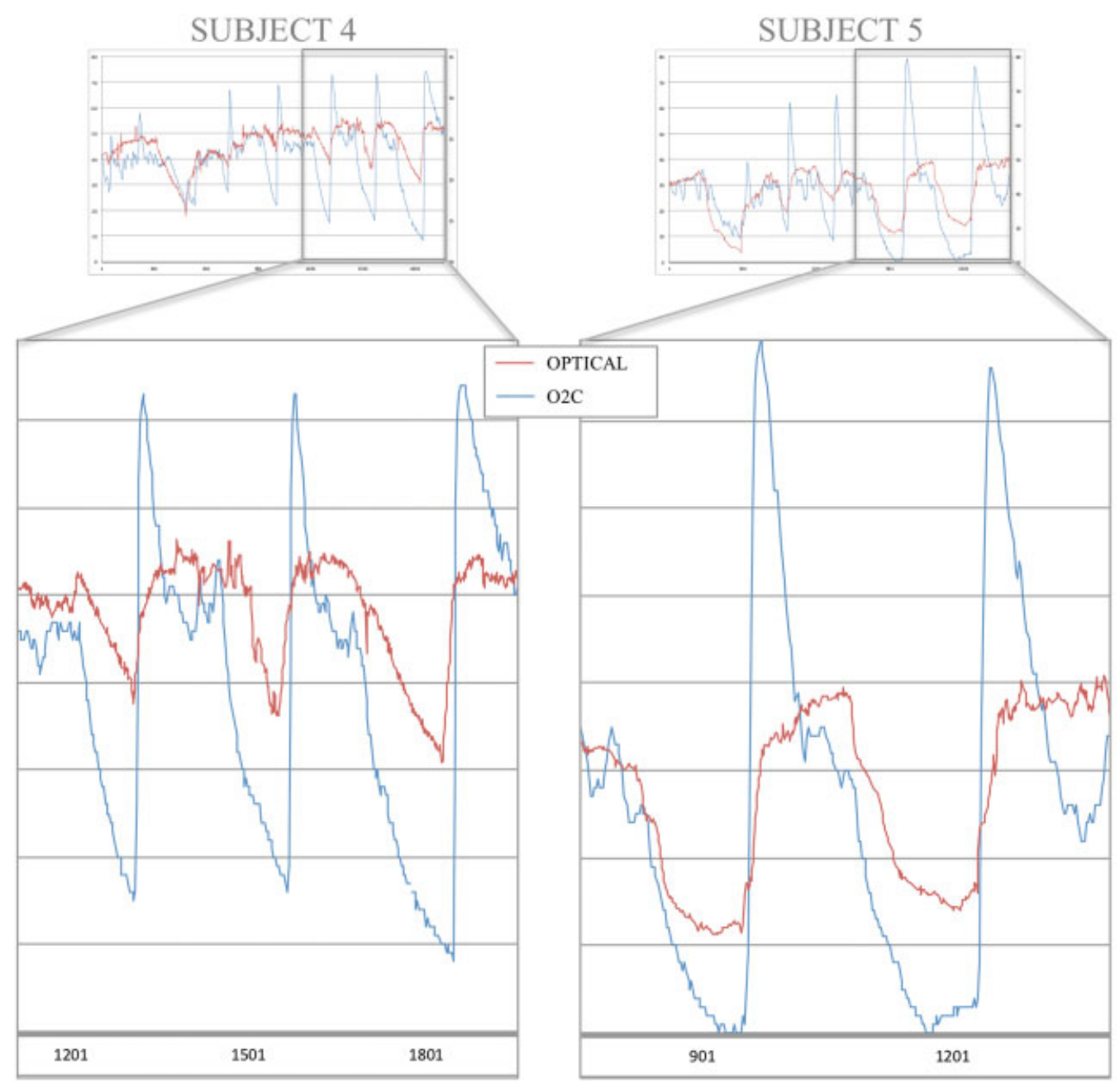

Fig. 7 Closer inspection of deoxygenation curves during cuff inflation shows intersubject variation (i.e., sharp trough vs. broad trough). These deoxygenation patterns are consistent between the two sensor modalities which provides a further indication of the validity of optical sensor measurements.

Also, in addition to accessing real-time oxygenation measurements, all data collected for each case will be available to the surgeon, facilitating trend analysis.

Free flap case series often report success rates of above $90 \% .{ }^{36}$ Cost-effectiveness figures from Poder et al, in which implantable Doppler technology was used to improve flap salvage rates, made the case that the cost of disposable monitoring equipment need be $£ 200$ or less in standard cases to become cost-neutral. Cost analyses show NIRS may be feasible with recent clinical case series, which saw reduced re-exploration and increased flap salvage rates from 57.7 to $96.6 \%{ }^{24}$ Reducing the upfront costs further would hopefully swing the cost-effectiveness in favor of continuous monitoring with an optical sensor.

Although the pressure cuff model was evaluated positively, it remains a nonclinical model and the sensor still requires further validation in either an animal model or clinical free flap series. The model simulated an "all-or-nothing" scenario, in which the vessel went from patent to occluded causing rapid deoxygenation. While this served its purpose for validation, slower and possibly fluctuating oxygenation levels caused by a transiently obstructed vessel or and expanding hematoma exerting external compressive forces may not be detected with as much confidence. Also, the sensor was in a static position during data collection which does not consider potential motion artifact in the clinical environment. To minimize data packet loss and battery restrictions the sensor was wired to the PC in this study. Further study is required to examine the wireless battery life and quality of data transfer. The $19.6 \times 12.4 \mathrm{~mm}$ circuit board is designed to incorporate another two boards in a stacked formation to provide battery and communication module (low energy Bluetooth). The sensor also requires housing in medical grade materials which block ambient light, are able to undergo sterilization, and can be fixed to the skin.

Regarding elevated tissue congestion during the AO model, it was hypothesized that the time taken to manually inflate the cuff (c.10 seconds) may have created a brief period of venous occlusion with arterial patency, causing the elevation. The extent of the elevation was significantly less than that during the VC model, maintaining a distinction between the simulations.

In practice, the technology underlying the sensor function will only work on those flaps with an accessible surface. As the sensor is designed with capabilities for wireless function, the electronics responsible for processing the signal and 


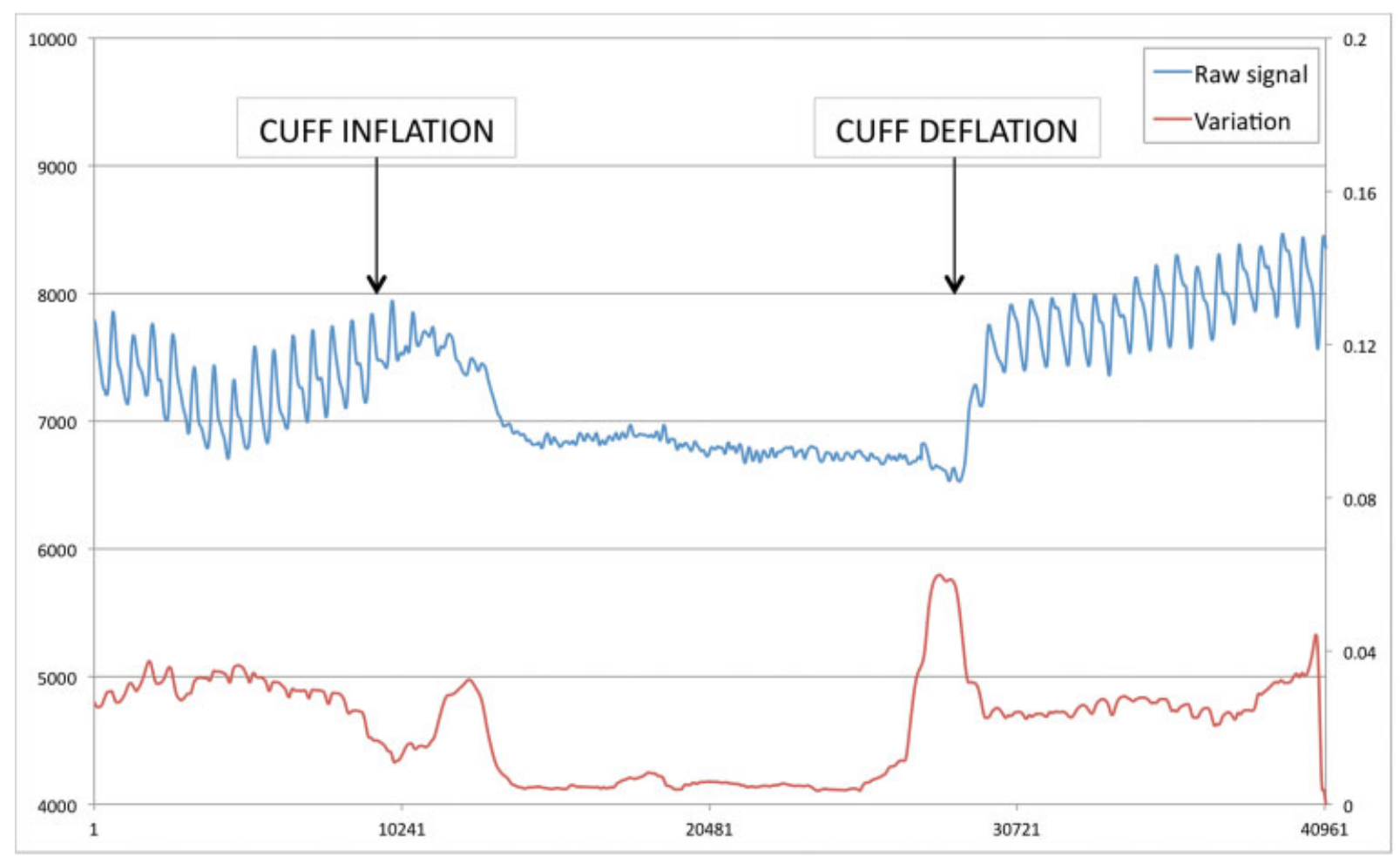

Fig. 8 An undulating sensor signal from the optical sensor (blue) confirms the presence of pulsatile flow, which stops during the arterial occlusion model and returns afterwards. The variance of the signal is calculated and shown as the red line (40-second sample, variation calculated over 2 seconds windows). Left axis, raw data signal; right axis - variation.

transmitting the data are all attached to the "probe." While this negates the need for a bedside computer and electric cabling, it adds some size to the parts in contact with the skin. As such, more inaccessible locations such as intraoral, perineal area, or those surfaces in close contact with the bed or chair are likely to be unsuitable. In these scenarios, specialized models with a short, wired connection between a thin interrogating probe and the remainder of the electronics are entirely feasible.

Implementing the sensor in the clinical setting initially need not immediately change practice, with information gathered primarily acting as supplementary to conventional monitoring strategies. As with all new technologies, there will be a learning curve before the full extent of benefit is realized, as shown in a NIRS free flap case series where there was a significantly decreased likelihood of surgical re-exploration in increments (odds ratio: 0.76; 95\% confidence interval: $0.62-0.94$ ) for every 100 flaps performed. ${ }^{24}$

\section{Conclusion}

This study provides encouraging data advocating the further development of low power, simplified wearable sensors for application in free flap monitoring. Results from previous clinical trials implementing similar flap monitoring technologies reveal improved patient outcomes and potential costsavings should the price of equipment remain low, which is only likely to be possible with technologies such as those described in this paper. In centers where flap success rates are particularly high, the proposed monitoring techniques may facilitate higher risk reconstructions and widen access to training opportunities.

\section{Authors' Contributions}

R.M.K., S.H., G.Z.Y., and A.D. conceptualized the study. C.M.C. and G.Z.Y. cooperated in hardware and software design. R.M.K., A.J.N., S.H., and A.D. performed the interpretation of data and clinical impact. All authors approved the manuscript.

\section{Funding}

This work was supported by an EPSRC grant (EP/H009744/ 1 ESPRIT).

Conflict of Interest

None declared.

\section{References}

1 Oni G, Malata CM. New surgical technique: Simultaneous use of contiguous intercostal spaces during total rib preservation exposure of the internal mammary vessels in microvascular breast reconstruction. J Plast Reconstr Aesthet Surg 2019;72(09): 1525-1529

2 Alt V, Donell ST, Chhabra A, Bentley A, Eicher A, Schnettler R. A health economic analysis of the use of rhBMP-2 in GustiloAnderson grade III open tibial fractures for the UK, Germany, and France. Injury 2009;40(12):1269-1275

3 Poder TG, Fortier PH. Implantable Doppler in monitoring free flaps: a cost-effectiveness analysis based on a systematic review of the literature. Eur Ann Otorhinolaryngol Head Neck Dis 2013; 130(02):79-85 
4 Che MP, Rozen WM, Whitaker IS, et al. Current evidence for postoperative monitoring of microvascular free flaps: a systematic review. Ann Plast Surg 2015;74(05):621-632

5 Genden EM, Rinaldo A, Suárez C, Wei WI, Bradley PJ, Ferlito A. Complications of free flap transfers for head and neck reconstruction following cancer resection. Oral Oncol 2004;40(10):979-984

6 Jandali S, Nelson JA, Sonnad SS, et al. Breast reconstruction with free tissue transfer from the abdomen in the morbidly obese. Plast Reconstr Surg 2011;127(06):2206-2213

7 Khouri RK, Cooley BC, Kunselman AR, et al. A prospective study of microvascular free-flap surgery and outcome. Plast Reconstr Surg 1998;102(03):711-721

8 Mangano DT, Layug EL, Wallace A, Tateo IMulticenter Study of Perioperative Ischemia Research Group. Effect of atenolol on mortality and cardiovascular morbidity after noncardiac surgery. N Engl J Med 1996;335(23):1713-1720

9 Moran SL, Illig KA, Green RM, Serletti JM. Free-tissue transfer in patients with peripheral vascular disease: a 10-year experience. Plast Reconstr Surg 2002;109(03):999-1006

10 Moran SL, Salgado CJ, Serletti JM. Free tissue transfer in patients with renal disease. Plast Reconstr Surg 2004;113(07):2006-2011

11 Shestak KC, Jones NF. Microsurgical free-tissue transfer in the elderly patient. Plast Reconstr Surg 1991;88(02):259-263

12 Siemionow M, Arslan E. Ischemia/reperfusion injury: a review in relation to free tissue transfers. Microsurgery 2004;24(06):468-475

13 Chen KT, Mardini S, Chuang DC, et al. Timing of presentation of the first signs of vascular compromise dictates the salvage outcome of free flap transfers. Plast Reconstr Surg 2007;120(01):187-195

14 Kääriäinen M, Halme E, Laranne J. Modern postoperative monitoring of free flaps. Curr Opin Otolaryngol Head Neck Surg 2018; 26(04):248-253

15 Karinja SJ, Lee BT. Advances in flap monitoring and impact of enhanced recovery protocols. J Surg Oncol 2018;118(05):758-767

16 Schmulder A, Gur E, Zaretski A. Eight-year experience of the CookSwartz Doppler in free-flap operations: microsurgical and reexploration results with regard to a wide spectrum of surgeries. Microsurgery 2011;31(01):1-6

17 Xipoleas G, Levine E, Silver L, Koch RM, Taub PJ. A survey of microvascular protocols for lower extremity free tissue transfer II: postoperative care. Ann Plast Surg 2008;61(03):280-284

18 Han ZF, Guo LL, Liu LB, et al. A comparison of the Cook-Swartz Doppler with conventional clinical methods for free flap monitoring: a systematic review and a meta-analysis. Int J Surg 2016; 32:109-115

19 Kohlert S, Quimby AE, Saman M, Ducic Y. Postoperative free-flap monitoring techniques. Semin Plast Surg 2019;33(01):13-16

20 Oda H, Beker L, Kaizawa Y, et al. A novel technology for free flap monitoring: pilot study of a wireless, biodegradable sensor. J Reconstr Microsurg 2020;36(03):182-190

21 Birkenfeld F, Naujokat H, Helmers AK, Purcz N, Möller B, Wiltfang J. Microdialysis in postoperative monitoring of microvascular free flaps: Experiences with a decision algorithm. J Craniomaxillofac Surg 2019;47(08):1306-1309
22 Kagaya Y, Miyamoto S. A systematic review of near-infrared spectroscopy in flap monitoring: Current basic and clinical evidence and prospects. J Plast Reconstr Aesthet Surg 2018;71(02): 246-257

23 Newton E, Butskiy O, Shadgan B, Prisman E, Anderson DW. Outcomes of free flap reconstructions with near-infrared spectroscopy (NIRS) monitoring: a systematic review. Microsurgery 2020; 40(02):268-275

24 Koolen PGL, Vargas CR, Ho OA, et al. Does increased experience with tissue oximetry monitoring in microsurgical breast reconstruction lead to decreased flap loss? the learning effect. Plast Reconstr Surg 2016;137(04):1093-1101

25 Creech B, Miller S. Evaluation of circulation in skin flaps. In: Grabb WC, Myers MB, eds. Skin Flaps. Boston: Little, Brown; 21-38

26 Chen C, Kwasnicki RM, Curto VF, Yang G, Lo BPL. Tissue oxygenation sensor and an active in vitro phantom for sensor validation. IEEE Sens J 2019;19(18):8233-8240

27 Chen CM, Kwasnicki R, Lo B, Yang GZWearable tissue oxygenation monitoring sensor and a forearm vascular phantom design for data validation. 11th International Conference on Wearable and Implantable Body Sensor Networks; June. 2014; Zurich, Switzerland. Accessed June 2014 at: https://ieeexplore.ieee.org/document/6855618

28 Wolff KD, Uekermann B, Matthes G, Wartenberg E. Intracapillary hemoglobin oxygenation and interstitial pO2 in venous flaps: an experimental study in rats. Microsurgery 1998;18(05):324-330

29 Rothenberger J, Amr A, Schaller HE, Rahmanian-Schwarz A. Evaluation of a non-invasive monitoring method for free flap breast reconstruction using laser doppler flowmetrie and tissue spectrophotometry. Microsurgery 2013;33(05):350-357

30 Plasswilm L, Tannapfel A, Cordes N, et al. Hypoxia-induced tumour cell migration in an in vivo chicken model. Pathobiology 2000;68(03):99-105

31 Brell B, Temmesfeld-Wollbrück B, Altzschner I, et al. Adrenomedullin reduces Staphylococcus aureus alpha-toxin-induced rat ileum microcirculatory damage. Crit Care Med 2005;33(04): 819-826

32 Knobloch K, Kraemer R, Lichtenberg A, et al. Achilles tendon and paratendon microcirculation in midportion and insertional tendinopathy in athletes. Am J Sports Med 2006;34(01):92-97

33 Beckert S, Witte MB, Königsrainer A, Coerper S. The impact of the micro-lightguide $\mathrm{O} 2 \mathrm{C}$ for the quantification of tissue ischemia in diabetic foot ulcers. Diabetes Care 2004;27(12):2863-2867

34 Steele MH. Three-year experience using near infrared spectroscopy tissue oximetry monitoring of free tissue transfers. Ann Plast Surg 2011;66(05):540-545

35 Lohman RF, Ozturk CN, Djohan R, Tang HR, Chen H, Bechtel KL. Predicting skin flap viability using a new intraoperative tissue oximetry sensor: a feasibility study in pigs. J Reconstr Microsurg 2014;30(06):405-412

36 Smit JM, Zeebregts CJ, Acosta R, Werker PM. Advancements in free flap monitoring in the last decade: a critical review. Plast Reconstr Surg 2010;125(01):177-185 